\title{
Comparison of a barium chloride test with ELISA for pregnancy detection in cows
}

\author{
Omer Ismaeel Dana ${ }^{1}$, Mnnat Talib Ghaidan ${ }^{1}$, \\ Rafiq Hamakarim Mukhtar ${ }^{2}$, Hiewa Othman Dyary ${ }^{1 \otimes}$ \\ ${ }^{1}$ College of Veterinary Medicine, University of Sulaimani, Sulaymaniyah 46001, Iraq \\ ${ }^{2}$ Directorate of Veterinary Services in Sulaymaniyah, Sulaymaniyah 46001, Iraq \\ dyary.othman@univsul.edu.iq
}

Received: March 26, $2020 \quad$ Accepted: October 5, 2020

\begin{abstract}
Introduction: Early detection of pregnancy is vital for appropriate reproductive management programmes to facilitate the rapid re-insemination of non-pregnant females and reduce the calving interval. Material and Methods: A barium chloride test was compared with a commercial progesterone ELISA to detect pregnancy in non-descriptive cows and investigate if it could be applied as an alternative to ELISA in the field. Blood and urine samples were collected from 74 cows with recorded insemination dates. The progesterone ELISA and barium chloride assay were implemented to detect progesterone (P4) in blood and urine specimens, respectively. The cows' reproductive systems were examined after they were slaughtered to determine the uterus's status. Macroscopic examination of the uterus was used as a reference standard for both tests. Results: The sensitivity rates of the P4 ELISA and barium chloride test to detect pregnant cows were $100.0 \%$ and $79.4 \%$, and to detect the corpus luteum (CL) were $83.0 \%$ and $87.0 \%$, respectively, their sensitivity increasing in the presence of the CL. The ELISA and barium chloride tests were $79.7 \%$ and $52.7 \%$ accurate in the diagnosis of pregnancy. The accuracy of the barium chloride test in CL detection increased to $81.0 \%$, and that of the ELISA to $86.4 \%$. There were no significant differences $(\mathrm{P}=0.052)$ between the barium chloride assay and ELISA when they were utilised for the identification of the CL. Conclusion: The barium chloride test can be an inexpensive and time-saving alternative to ELISA in pregnancy diagnosis when the insemination date is known.
\end{abstract}

Keywords: barium chloride, cows, corpus luteum, pregnancy diagnosis, progesterone.

\section{Introduction}

Humans have attempted since the early civilisations to determine the pregnancy status of cattle after parturition. For instance, between 2200 and 2100 BC, the ancient Egyptians noticed that the germination and growth of wheat shoots and barley seeds were inhibited by the urine of pregnant cows (6). Researchers continue attempting to find inexpensive, accurate, and practical methods that can detect pregnancy in cattle in a short time (13).

The capability for early and accurate pregnancy detection in cows is crucial for better reproductive management programmes (20). Early pregnancy detection allows the re-insemination of non-pregnant cows as soon as possible to reduce calving intervals and replace infertile cows. Accurate and early detection of pregnancy is a necessary procedure in livestock management since it assists in reducing open days of cows that failed to conceive, leading to significant economic gains (17). When a pregnant cow is mistakenly classified as non-pregnant, the stresses caused by treatment and re-insemination might increase the possibility of iatrogenic abortion (11).

Adequate post-insemination concentrations of progesterone ( $\mathrm{P} 4)$ are required for successful pregnancy in cows (12). P4 is a steroid hormone secreted by the corpus luteum (CL) in the non-pregnant female, and by the placenta and $\mathrm{CL}$ in the pregnant female. It inhibits reproductive behaviours and assists in the maintenance of pregnancy (1). The CL graviditatis keeps the P4 concentration at a high level during the gestation period, but regression of the CL causes the level of $\mathrm{P} 4$ to fall rapidly just before the onset of delivery (24). Endometrial secretion, increasing the level of progesterone during pregnancy, provides a suitable environment for the conceptus in the uterus necessary for it to survive. P4 prevents abortion by inducing quiescence and noncontractility in the myometrium (10). 
The hormonal method, which depends on progesterone determination in blood, is considered one of the methods of pregnancy detection in cows effective as early as 19-21 days after insemination (9). Presently, several diagnostic techniques are in existence and are frequently employed on the farm. Traditional methods of pregnancy diagnosis in cattle, such as rectal palpation or ultrasonography, are commonly used. With palpation per rectum, the accuracy earlier than at 30-35 days of gestation can be low, and the method risks inducing abortion during examination (18). Trans-rectal ultrasonography is commonly applied for pregnancy detection, but it requires the skills of specialised personnel (2). Pregnancy can also be recognised through the detection of progesterone in the serum by ELISA (19). Other methods of ascertaining pregnancy depend on the detection of progesterone metabolic end-products in urine.

The goal of this research was to evaluate the accuracy of two different methods of pregnancy detection in cows; namely, the progesterone ELISA and barium chloride test. The barium chloride method can be easily applied in the field and does not need a specific laboratory. In this method, samples can be tested easily by mixing a small amount of urine with barium chloride, and the result will appear in just a few minutes (8). However, the accuracy of this technique needs to be investigated in a standardised study. Hence, the present research was conducted to compare a barium chloride test with a commercial progesterone ELISA for pregnancy diagnosis in cows. A macroscopic examination of the uterus was used as a gold standard to confirm both tests' efficacy. This comparison clarifies if barium chloride could be a less costly and time-saving alternative method for pregnancy diagnosis.

\section{Material and Methods}

Study animals and sample collection. The research was carried out between July and October 2018 at the Sulaimani abattoir in the city of Sulaymaniyah, Iraq. Seventy-four cows of varying ages, parities, and breeds which were brought to the abattoir for slaughter were selected to be included in the study. Records of the cows' insemination accompanied the animals. Before the slaughter of each cow, blood was withdrawn from the coccygeal vein and put into a plain test tube. Blood samples were transported to the laboratory in a coolbox with ice packs within six hours. Upon arrival at the laboratory, the samples were centrifuged at 2,000 $\times g$ for $10 \mathrm{~min}$ to separate the serum, which was stored at $-20^{\circ} \mathrm{C}$ until hormonal analysis for progesterone was performed.

After the cow was slaughtered, about $20 \mathrm{~mL}$ of urine was collected directly from the urinary bladder by syringe for the barium chloride test.

Determination of pregnancy. Progesterone concentration $(\mathrm{ng} / \mathrm{mL})$ was determined by a commercial competitive ELISA (Monobind Inc., USA). The kit contained 96-well ELISA plates and the essential reagents for blood sample analysis. The standard curve ranged from 0 to $60 \mathrm{ng} / \mathrm{mL}$. An ELx800 absorbance microplate reader (BioTek, USA) was utilised to measure the absorbance at $450 \mathrm{~nm}$ (reference wavelength $620 \mathrm{~nm}$ ). The outcomes were calculated on a standard curve, which was plotted using a linear regression equation. Five millilitres of urine was put into a test tube and mixed with 5 or 6 drops of $1.0 \%$ barium chloride. The appearance of white-coloured precipitation indicated that the cow was non-pregnant. In contrast, a lack of precipitation was an indicator of pregnancy (16).

After the cows were slaughtered, the uteruses were examined to determine the status of pregnancy. We used the gross examination of the reproductive system as the standard reference for both tests.

Statistical analysis. All the statistical analyses of the data and 2-D dot plots presented in Fig. 1 were performed with the SPSS software package version 22.0 (IBM SPSS, USA). The level of significance $\mathrm{P}<0.05$ was established for all statistical analyses. Results were analysed by chi-squared test evaluating the accuracy of both techniques for detecting pregnancy and the CL in cows. Data collected were compared to the results of the gross examination of the uteruses. The accuracy, specificity, and sensitivity of both techniques were derived. The results were categorised as correct negative $(\mathrm{CN})$, correct positive $(\mathrm{CP})$, false negative $(\mathrm{FN})$, and false-positive (FP). From these values, the accuracy, specificity, and sensitivity of both techniques were extrapolated. Accuracy was calculated as $100 \times((\mathrm{CP}+$ $\mathrm{CN}) \div(\mathrm{CN}+\mathrm{CP}+\mathrm{FP}+\mathrm{FN}))$, sensitivity as $100 \times(\mathrm{CP}$ $\div(\mathrm{CP}+\mathrm{FN}))$, and specificity as $100 \times(\mathrm{CN} \div(\mathrm{CN}+\mathrm{FP}))$. The positive predictive value (PPV) was calculated as $100 \times(\mathrm{CP} \div(\mathrm{CP}+\mathrm{FP}))$ and the negative predictive value $(\mathrm{NPV})$ as $100 \times(\mathrm{CN} \div(\mathrm{CN}+\mathrm{FN}))(7)$.

\section{Results}

The data from Table 1 show the numbers of results grouped as correct positive, correct negative, false positive, and false negative for pregnancy assessment using the progesterone ELISA and barium chloride tests. These assumptions were based upon the macroscopic examination of the genital tracts. The progesterone ELISA results indicated that 34 of the 74 cows $(45.9 \%)$ were diagnosed correctly as pregnant, and $25(33.8 \%)$ as non-pregnant. However, using the barium chloride test, $27(36.5 \%)$ cows were correctly identified as pregnant and $12(16.2 \%)$ as non-pregnant. Fifteen $(20.3 \%)$ falsepositive diagnoses were recorded with the progesterone ELISA, but the barium chloride test resulted in 28 (37.8\%) false-positive and $7(9.5 \%)$ false-negative diagnoses. The accuracy of the progesterone ELISA and barium chloride test for each different reproductive state of the cows is illustrated in Fig. 1. 
The accuracy, sensitivity, specificity, PPV, and NPV of both tests are given in Table 2 . The progesterone ELISA was $100.0 \%$ sensitive in the detection of pregnancy and $83.0 \%$ sensitive in the detection of the CL. However, the sensitivity of the barium chloride test for identification of pregnancy did not exceed $79.4 \%$, while for the CL it reached $87.0 \%$. The progesterone ELISA was $62.5 \%$ specific, and the barium chloride test
$30.0 \%$ specific in pregnancy detection. In a marked difference, the tests were respectively $100.0 \%$ and $65.0 \%$ specific in the identification of the CL. The identification of pregnancy by the progesterone ELISA was $79.7 \%$ accurate, while the barium chloride test was $52.7 \%$ accurate in this application. However, both techniques were close in their CL-detection accuracy, achieving $86.4 \%$ and $81.0 \%$, respectively.

Table 1. Correct positive, correct negative, false positive, and false-negative results by progesterone ELISA and barium chloride tests for detection of pregnancy and corpus luteum in cows

\begin{tabular}{lllllllll}
\hline & \multicolumn{3}{c}{ Progesterone ELISA (number (\%)) } & \multicolumn{3}{c}{ Barium chloride test (number (\%)) } \\
\cline { 2 - 7 } & $\begin{array}{l}\text { Correct } \\
\text { positive }\end{array}$ & $\begin{array}{l}\text { Correct } \\
\text { negative }\end{array}$ & $\begin{array}{l}\text { False- } \\
\text { positive }\end{array}$ & $\begin{array}{l}\text { False- } \\
\text { negative }\end{array}$ & $\begin{array}{l}\text { Correct } \\
\text { positive }\end{array}$ & $\begin{array}{l}\text { Correct } \\
\text { negative }\end{array}$ & $\begin{array}{l}\text { False- } \\
\text { positive }\end{array}$ & $\begin{array}{l}\text { False- } \\
\text { negative }\end{array}$ \\
\hline Pregnancy & $34(45.9)$ & $25(33.8)$ & $15(20.3)$ & 0 & $27(36.5)$ & $12(16.2)$ & $28(37.8)$ & $7(9.5)$ \\
CL & $49(66.2)$ & $15(20.3)$ & 0 & $10(13.5)$ & $47(63.5)$ & $13(17.6)$ & $7(9.5)$ & $7(9.5)$ \\
\hline
\end{tabular}

There were significant differences (0.00) between the techniques, but the difference was not significant (0.52) for CL identification. CL - corpus luteum. Test: chi-squared

Table 2. Values of sensitivity, specificity, positive predictive value (PPV), negative predictive value (NPV), and accuracy of progesterone ELISAand barium chloride tests for detection of pregnancy and corpus luteum

\begin{tabular}{|c|c|c|c|c|c|c|}
\hline Method of detection & & Sensitivity & Specificity & PPV & NPV & Accuracy \\
\hline \multirow[t]{2}{*}{ Progesterone ELISA } & Pregnancy & 100.0 & 62.5 & 69.4 & 100.0 & 79.7 \\
\hline & $\mathrm{CL}$ & 83.0 & 100.0 & 100.0 & 60.0 & 86.4 \\
\hline \multirow[t]{2}{*}{ Barium chloride test } & Pregnancy & 79.4 & 30.0 & 49.0 & 63.0 & 52.7 \\
\hline & $\mathrm{CL}$ & 87.0 & 65.0 & 87.0 & 65.0 & 81.0 \\
\hline
\end{tabular}

$\mathrm{CL}$ - corpus luteum; PPV - positive predictive value; NPV - negative predictive value

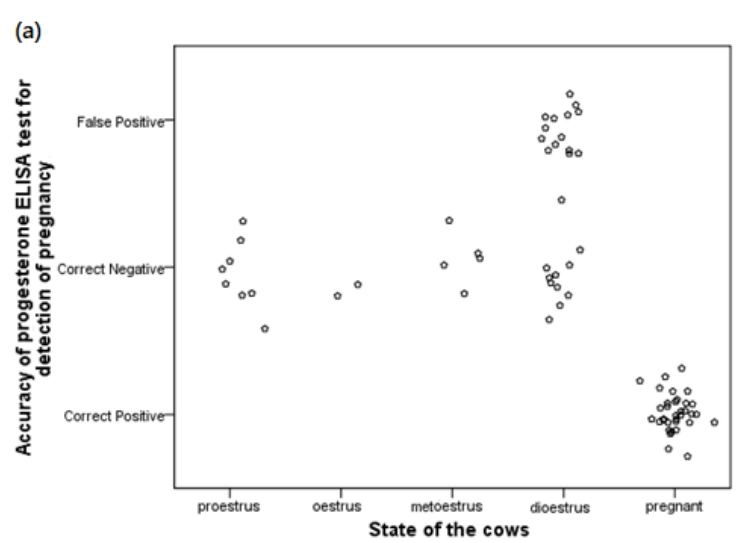

(c)

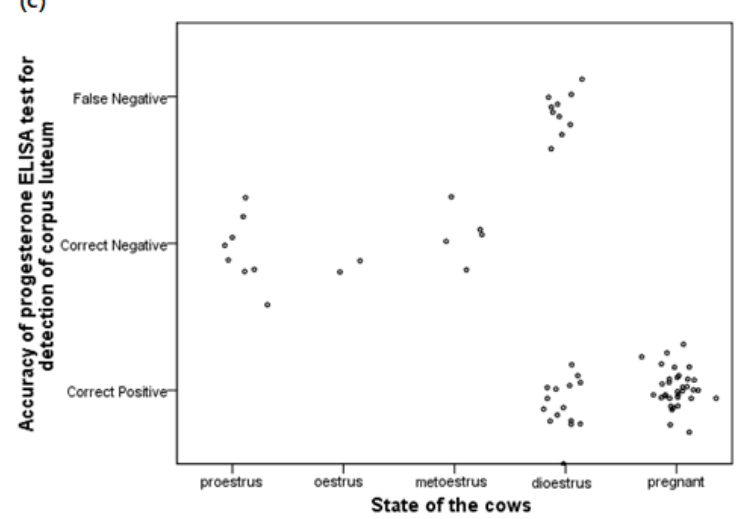

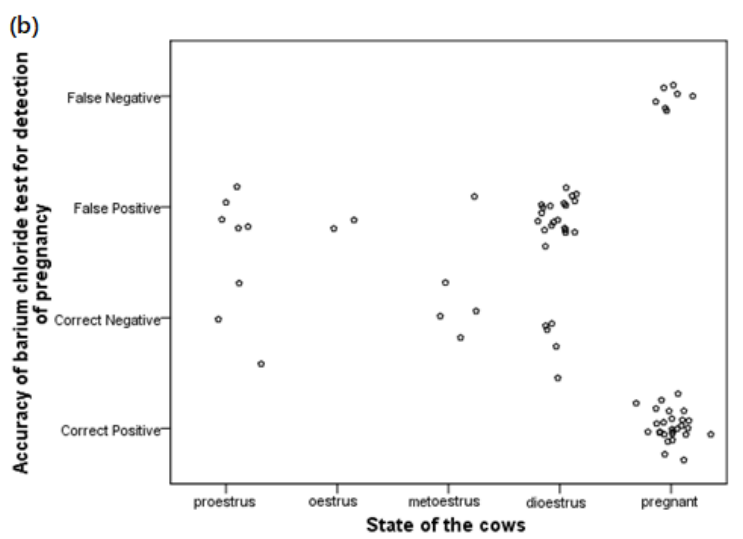

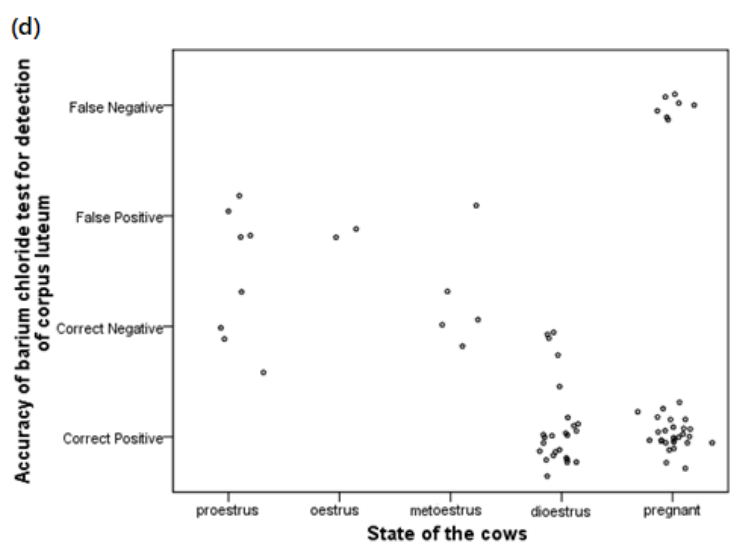

Fig. 1. Scatter plots showing result quality by phase in the oestrous cycle. Plots (a) and (b) show the accuracy of the progesterone ELISA and barium tests for pregnancy detection, while (c) and (d) show the accuracy of the tests for CL detection 


\section{Discussion}

Undoubtedly, the identification of non-pregnant cows is vital to decrease the time between calving and establishing a subsequent pregnancy, thereby improving milk production and generating more income for farmers.

Many studies have focused on the detection of pregnancy by determining progesterone levels via blood, milk, and faecal samples using ELISA and radioimmunoassay techniques (19). These methods require radioisotopes and the cooperation of appropriate laboratories for the results to be read. These circumstances are not necessary for pregnancy diagnosis by the barium chloride test, which provides the simultaneous examination of many samples in a short time and within the restrictions of field conditions. The liver transforms progesterone into the inactive metabolite pregnanediol glucosiduronidate, which is excreted in the urine (21). In the barium chloride test, urine samples can be used for the detection of this residue of progesterone (8).

The macroscopic examination of uteruses after the slaughter of the cows was depended upon as a reference (gold) standard. Other researchers also used this method as a standard to compare different pregnancy diagnostic methods (25), since the probability of it falsely diagnosing pregnancy is $0.0 \%$. Other methods of pregnancy detection might not be as accurate; for example, the accuracy of ultrasound might reach $93.7 \%$ to $97.8 \%$ on day 27 (22).

The sensitivity of the progesterone ELISA for pregnancy diagnosis in the present investigation was $100.0 \%$ and decreased to $83.0 \%$ when used for CL identification. This outcome is consistent with that recorded by Inaudi et al. (4), who found $84.8 \%$ for pregnancy. In contrast, other researchers recorded $84.8 \%$ (3), which was lower than the outcomes obtained in the current study. This outcome might be due to a persistent CL or silent heat, or early embryonic death (4). In this study, the sensitivity of the barium chloride test for pregnancy detection was measured at $79.4 \%$, and for CL detection at $87.0 \%$.

The specificity of the progesterone ELISA for the identification of non-pregnant cattle in the present investigation was $62.5 \%$. This was lower than the specificity of this test for the detection of the CL, which reached $100 \%$. The barium chloride test was also more specific in the detection of the CL than in pregnancy diagnosis, where $65.0 \%$ and $30.0 \%$ were seen, respectively. This difference is because the CL secretes $\mathrm{P} 4$, which is present in pregnant and non-pregnant cows (5). Hence, P4 is not unique to pregnancy.

The accuracy of the progesterone ELISA in the detection of pregnancy was not any higher than $79.7 \%$. Our results are lower than the $87.0 \%$ reported by Samsonova et al. (19), and $84.8 \%$ by Ghaidan et al. (3). Pennington et al. (15) noted increasing accuracy rates from $85.4 \%$ to $88.4 \%$ from day 21 to day 24 after artificial insemination. This result is nearly equivalent to our results when the progesterone ELISA was used for CL identification, for which $86.6 \%$ accuracy was attained.

The inconsistencies in the accuracies of the tests to detect pregnancy and the CL are clarified in Fig. 1. The number of false-positive results increased when the progesterone ELISA was used for pregnancy detection. Fifteen samples $(20.2 \%)$ were taken from cows in the dioestrous phase of the oestrous cycle, and the CL was present in those cows, which increases progesterone level in the circulating blood and will raise the rate of false pregnancy diagnosis. Accuracy is also affected by the technique used for the diagnosis of pregnancy; the accuracy by ELISA is approximately $75.8 \%$, while by radioimmunoassay it is about $80.5 \%$ (14).

The barium chloride test in the current study was $52.7 \%$ accurate in pregnancy detection and $81.0 \%$ in CL detection. This variation of accuracy is due to a persistent CL, which increases false-positive results during pregnancy diagnosis. Also, high feed intake by cows increases the metabolism of progesterone (23), which leads to increased concentration of pregnanediol glucosiduronidate in the urine and increased falsepositive results. Besides this metabolite, exogenous sources of oestrogens in the diet may lead to false-positive diagnoses. However, low $\mathrm{P} 4$ production during pregnancy will also increase the false-negative results (5).

In this investigation, there were no significant differences ( $\mathrm{P}=0.052)$ between the techniques for the identification of the corpus luteum (Table 1). Thus, the outcomes of the current study indicated that the barium chloride test could be used instead of the progesterone ELISA if some constraints are borne in mind.

In conclusion, the present investigation confirmed the progesterone ELISA and barium chloride test to be reliable and convenient for pregnancy diagnosing in urine and plasma samples if collected from those cows which have recorded insemination data. The outcomes of this research confirm that the progesterone ELISA is a more accurate method than the barium chloride test to detect pregnancy. However, the accuracy of both tests increases when the history of insemination is recorded. Given these considerations, the barium chloride test may be considered an excellent alternative to the progesterone ELISA to detect pregnancy in the field, provided the history of insemination is known.

Conflict of Interest Statement: The authors declare that there is no conflict of interests regarding the publication of this article.

Animal Rights Statement: All procedures that include animals were approved by the Animal Care and Use Committee at the University of Sulaimani through approval number AUP-2018-05.

Financial Disclosure Statement: The University of Sulaimani provided the funds to conduct this study. 


\section{References}

1. Couto S.R., Guerson Y.B., Ferreira J.E., Silva O.R., Silenciato L.N., Barbero R.P., Mello M.R.: Impact of supplementation with longacting progesterone on gestational loss in Nelore females submitted to TAI. Theriogenology 2019, 125, 168-172.

2. Fricke P.: Scanning the future-Ultrasonography as a reproductive management tool for dairy cattle. J Dairy Sci 2002, 85, 1918-1926.

3. Ghaidan M., Dana O., Dyary H.: Accuracy of bovine pregnancyassociated glycoproteins (bPAGs) in the diagnosis of pregnancy: a comparative study of three pregnancy diagnostic methods. Pol J Vet Sci 2019, 22, 769-775.

4. Inaudi P., Bacigalupo M., Monittola C., Lugaro G., Genazzani A.: Pregnancy diagnosis in cattle by a rapid and highly reliable method for progesterone determination in milk. Reproduction 1982, 65, 265-273.

5. Jaimes L., Vinet R., Knox M., Morales B., Benites J., Laurido C., Martínez J.L.: A review of the actions of endogenous and exogenous vasoactive substances during the estrous cycle and pregnancy in rats. Animals 2019, 9, 288.

6. Juodžentytė R., Žilaitis V.: Practical evaluation pregnancy of heifers by punyakoti test. Vet Zootech-Lith 2016, 74, 14-17.

7. Karen A.M., Darwish S., Ramoun A., Tawfeek K., Van Hanh N., de Sousa N.M., Sulon J., Szenci O., Beckers J.-F.: Accuracy of transrectal palpation for early pregnancy diagnosis in Egyptian buffaloes. Trop Anim Health Prod 2011, 43, 5-7.

8. Kubátová A., Fedorova T., Skálová I., Hyniová L.: Non-invasive pregnancy diagnosis from urine by the Cuboni reaction and the barium chloride test in donkeys (Equus asinus) and alpacas (Vicugna pacos). Pol J Vet Sci 2016, 19, 477-484.

9. Laing J., Heap R.: The concentration of progesterone in the milk of cows during the reproductive cycle. Br Vet J 1971, 127, 14-22.

10. Lewis G.: Role of ovarian progesterone and potential role of prostaglandin F2 $\alpha$ and prostaglandin E2 in modulating the uterine response to infectious bacteria in postpartum ewes. J Anim Sci 2003, 81, 285-293.

11. Lucy M.: Reproductive loss in high-producing dairy cattle: where will it end? J Dairy Sci 2001, 84, 1277-1293.

12. Mann G., Lamming G.: The influence of progesterone during early pregnancy in cattle. Reprod Domest Anim 1999, 34, 269-274.

13. Moussafir Z., Allai L., El Khalil K., Essamadi A., El Amiri B.: Could a bovine pregnancy rapid test be an alternative to a commercial pregnancy-associated glycoprotein ELISA test in dairy cattle? Anim Reprod Sci 2018, 192, 78-83.
14. Nebel R., Whittier W., Cassell B., Britt J.: Comparison of on-farm and laboratory milk progesterone assays for identifying errors in detection of estrus and diagnosis of pregnancy. J Dairy Sci 1987, 70, 1471-1476.

15. Pennington J., Schultz L., Hoffman W.: Comparison of pregnancy diagnosis by milk progesterone on day 21 and day 24 postbreeding: Field study in dairy cattle. J Dairy Sci 1985, 68, 2740-2745.

16. Rao S., Veena T.: Comparison of seed germination test with urine barium chloride test and milk copper sulphate test for efficacy to detect pregnancy in cows. Indian J Anim Res 2009, 43, 124-126.

17. Reese S., Pereira M., Vasconcelos J., Smith M., Green J., Geary T., Peres R., Perry G., Pohler K.: Markers of pregnancy: how early can we detect pregnancies in cattle using pregnancyassociated glycoproteins (PAGs) and microRNAs? Anim Reprod 2018, 13, 200-208

18. Romano J.E., Bryan K., Ramos R.S., Velez J., Pinedo P.: Effect of early pregnancy diagnosis by per rectum amniotic sac palpation on pregnancy loss, calving rates, and abnormalities in newborn dairy calves. Theriogenology 2016, 85, 419-427.

19. Samsonova J.V., Osipov A.P., Kondakov S.E.: A new dried milk sampling technique and its application for progesterone detection in cows. Vet J 2014, 199, 471-472.

20. Schanzenbach C.I., Kirchner B., Ulbrich S.E., Pfaffl M.W.: Can milk cell or skim milk miRNAs be used as biomarkers for early pregnancy detection in cattle? PloS One 2017, 12, e0172220, doi: 10,1371/journal.pone.0172220.

21. Schiffer L., Barnard L., Baranowski E.S., Gilligan L.C., Taylor A.E., Arlt W., Shackleton C.H., Storbeck K.-H.: Human steroid biosynthesis, metabolism and excretion are differentially reflected by serum and urine steroid metabolomes: a comprehensive review. J Steroid Biochem Mol Biol 2019, 194, 105439, doi: 10.1016/j.jsbmb.2019.105439.

22. Silva E., Sterry R., Kolb D., Mathialagan N., McGrath M., Ballam J., Fricke P.: Accuracy of a pregnancy-associated glycoprotein ELISA to determine pregnancy status of lactating dairy cows twenty-seven days after timed artificial insemination. J Dairy Sci 2007, 90, 4612-4622.

23. Vasconcelos J.L.M., Sangsritavong S., Tsai S.-J., Wiltbank M.: Acute reduction in serum progesterone concentrations after feed intake in dairy cows. Theriogenology 2003, 60, 795-807.

24. Yamada O., Abe M., Takehana K., Iwasa K., Hiraga T., Hiratsuka T.: Microvascular changes in the bovine corpus luteum during pregnancy. J Vet Med Sci 1995, 57, 75-79.

25. Žvorc Z., Matijatko V., Beer B., Foršek J., Bedrica L., Kučer N.: Blood serum proteinograms in pregnant and non-pregnant cows. Vet Arhiv 2000, 70, 21-30. 